\title{
Case Report: Molar Intrusion with Temporary Anchorage Device
}

\author{
Israel $K^{1}$, González $\mathbf{N}^{1}$ and Ximena Toledo Pinto ${ }^{2 *}$ \\ ${ }^{1}$ Dental Surgeon, Universidad Andrés Bello, Chile \\ ${ }^{2}$ Assistant Professor, Universidad de Chile, Chile
}

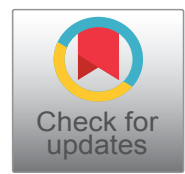

*Corresponding author: Ximena Toledo Pinto, Dental Surgeon, Specialist in Orthodontics, Assistant Professor, Universidad de Chile, Chile

\begin{abstract}
A clinical case report of the $2 \mathrm{~mm}$. intrusion of tooth 1.6 and the rehabilitation of the space left by the extracted tooth 4.6 with a single implant and crown.

Four micro screws were installed in relation to tooth 1.6, two buccal and two in the hard palate. The tooth was intruded in a period of six months with two power chains anchored to cross-shaped micro screws. The chains were replaced every 21 days. The implant to replace tooth 4.6 was also placed and after six months tooth 1.6 was in position and the implant was osseointegrated and ready to be rehabilitated.

The objectives, function and esthetics, were achieved without the need of occlusal wear nor the loss of tooth vitality. Four years after treatment the case is stable, without changes.
\end{abstract}

\section{Keywords}

Intrusion, Anchorage, Micro screw

\section{Introduction}

Orthodontic's primary objective is to correctly align teeth, restore esthetics and function, so that the orofacial health of each patient is in harmony.

When patients come to us looking for esthetics or function, we find ourselves faced with different problems, in tooth position, missing teeth, tooth size, etc.; in this case report our focus was on tooth position.

There are several reasons why tooth loss occurs and cavities are the most common one. When this happens, we must arrange the teeth without considering the missing one or if necessary, consider it and look for the best option to replace it.

There are cases when a missing tooth will affect the antagonistic one. It extrudes and results in a discrepan$\mathrm{cy}$ in the occlusal plane. This is when oral rehabilitation experts step in and solve the occlusal problem by treating the extruded tooth by wearing down the enamel or through an inlay or crown.

Nowadays there are other much less invasive techniques such as intruding a tooth orthodontically by using micro screws which avoids any dental wear.

Micro screws are metallic devices that serve as orthodontic anchorage and are composed of stainless steel and titanium. Their dimensions vary and in diameter go from 1.3-2 mm. and in length from 6-12 $\mathrm{mm}$, with different head shapes.

They allow us to perform different movements on the teeth and are easy to place and remove. Among its main functions are molar intrusion, space closure, midline correction, etc. They are generally placed in the posterior buccal and palatal areas of the mouth or over the smile line so as to not affect the patient's esthetics.

In order to place the micro screws accurately, precise planning with a cone- beam must be done so we have information on the density of the bone, anatomical references and we can then define the length and angulation of the micro screw to be used.

This case report shows us a therapeutical alternative to what is usually done, which is to wear down the extruded tooth to the point of it losing its vitality and then rehabilitating the lower segment.

\section{Case Report}

Medical record and exam: Female patient, 35 years old. ASA I. 
Chief complaint: Complete mouth restauration. The initial clinical examination indicated the presence of several restorations, tooth 4.6 missing for four years and extrusion of tooth 1.6, invading the occlusal space.

Other than tooth 1.6 , the occlusion is stable.

The periodontal examination did not reveal any problems. PSR (Periodontal Screening and Recording) was no higher than two and she had excellent oral hygiene.

\section{Treatment planning}

The functional findings revealed a $2 \mathrm{~mm}$. extrusion of tooth 1.6 and gingival recession due to the early loss of tooth 4.6. A conservative treatment was planned involving Orthodontics, Oral Rehabilitation and Implantology.

Four micro screws were placed after the $\mathrm{X}$-rays were studied, two on the buccal and two on the hard palate in relation to tooth 1.6. The micro screws were placed as anchorage in order to intrude it and the missing tooth 4.6 would be replaced by an implant and crown.

In addition a digital plan was made to determine what we would achieve.

\section{Step by step}

The cone-beam was properly studied in order to know all the relevant parameters for placing the micro screws and the implant in the lower arch.

The functional findings revealed a $2 \mathrm{~mm}$. extrusion of

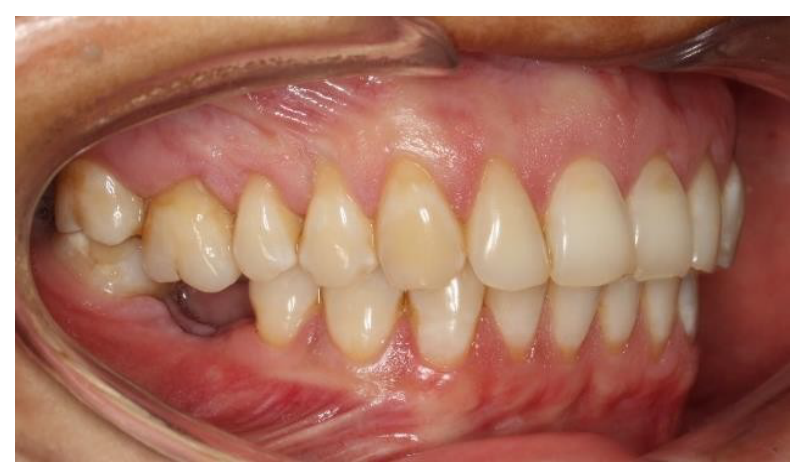

Figure 1: Extrusion of piece 1.6.

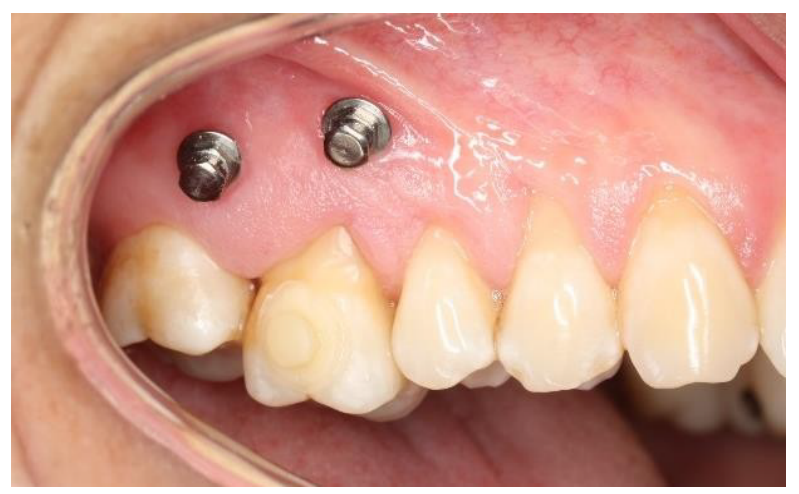

Figure 2: Installed vestibular TADs. tooth 1.6 and gingival recession due to the early loss of tooth 4.6 (Figure 1).

Using Ortho System software, the amount of intrusion necessary in order to rehabilitate the lower arch was determined.

Four Dewimed MOSAS micro screws were placed, two on the buccal ( $7 \mathrm{~mm}$ long/1.3 diameter) and two on the hard palate $(9 \mathrm{~mm}$ long/ 1.3 diameter) in relation to tooth 1.6. The exact site was determined after studying the cone-beam and where it would be most effective to intrude the molar.

To replace tooth 4.6 a BioHorizons Cylindrical Tapered Internal $4.6 \times 12 \mathrm{~mm}$. implant was chosen (Figure 2).

Two continuous power chains anchored on the micro screws were placed, in a criss-crossed fashion over the occlusal face of the molar applying 50 gr. of force measured with a PCE-LFG 10 dynamometer. The intrusion was done in an axial direction. Every 21 days the power chains were replaced by new ones and always set in position with composite (Figure 3).

In six months the tooth was intruded and for the following six months it was held in retention by replacing the power chain, in the same fashion, with 0.012 " metallic ligatures.

While tooth 1.6 was in retention, the implant of

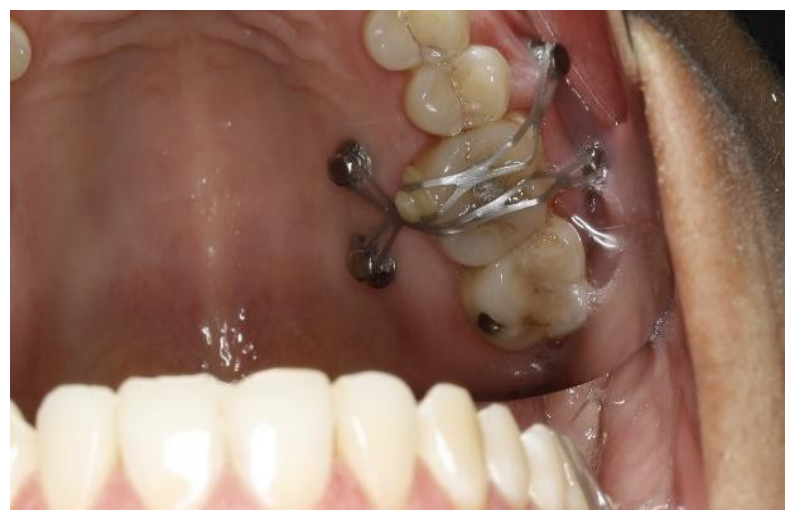

Figure 3: Intrusion mechanism in piece 1.6.

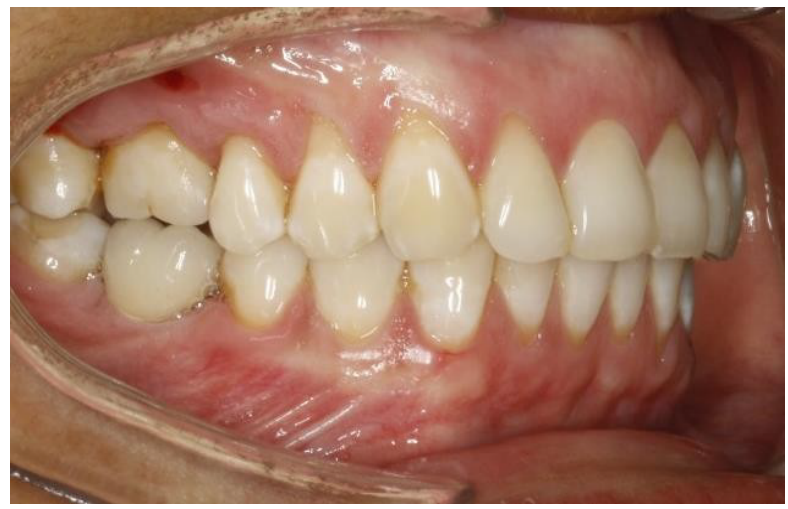

Figure 4: Treatment finished. 
tooth 4.6 was installed and in process of osseointegration. This helped in making our treatment more efficient.

In six months tooth 1.6 was correctly positioned on the occlusal plane and the lower implant was in optimal condition to be rehabilitated. The crown was set on tooth 4.6 and the upper metallic retention and the micro screws were removed.

The stability of the treatment was accomplished by having both areas working together, implantology and rehabilitation. The intrusion of tooth 1.6 and the implant replacing tooth 4.6 will allow for the treatment to last in time, preventing any new changes in position (Figure 4).

\section{Discussion and Conclusion}

Nowadays micro screws have a very important role in orthodontic treatments since they allow for individual tooth movement without affecting neighbouring teeth, eliminating Newton's third law.

The greatest advantage of using these micro screws is that it allows us to preserve the health of the tooth considering that in this clinical case the original treatment plan was to submit it to endodontic treatment and then rehabilitate it with a crown.

They are biocompatible and immediate forces can be applied to them; due to their small size, they can be placed in interradicular spaces and better results are obtained when compared to conventional treatments. The surgical technique is simple and not as invasive; the instrumentation used is basic and does not require costly nor sophisticated materials.

It is a comfortable treatment option for the patient since it is painless and non-inflammatory. The results were a success, conservative and stable in time.

The treatment was planned with a conservative approach; the priority must always be to maintain the tooth's vitality and avoid its extraction; in our clinical case tooth 1.6 has a vital role as described in the six keys of normal occlusion.

At first the plan was to treat tooth 1.6 with a crown which not only forces us to devitalize the tooth but also diminishes the prognosis and longevity of the tooth in question; in the best scenario a crown will last 20 years but with a micro screw the tooth is preserved forever as long as the patient has a proper daily oral hygiene. The intrusion of tooth 1.6 and implant replacing 4.6 assures us stability and longevity of the treatment [1-6].

\section{References}

1. Gutiérrez Labaye $P$, Hernández Villena R, Perea García MA, Escudero Castaño N, Bascones Martínez A (2014) Microtornillos: Una revisión. Av Periodon Implantol 1: 25-38.

2. Kravitz ND, Kusnoto B, Tsay TP, Hohlt WF (2007) The use of temporary anchorage devices for molar intrusion. $\mathrm{J} \mathrm{Am}$ Dent Assoc 138: 56-64.

3. Kamlesh Singh, Deepak Kumar, Raj Kumar Jaiswal, Amol Bansal (2010) Temporary anchorage devices-miniimplants. Natl J Maxillofac Surg 1: 30-34.

4. Paik CH, Park IK, Y Woo, TW Kim (2009) Orthodontic miniscrew implants. Mosby/Elsevier, 175-185.

5. Cheol-Ho Paik (2013) Molar intrusion using TADs: The key element to correcting anterior open bite and/or vertical excess problems. AAO Annual Session, Philadelphia.

6. Curiel-Meza BY, Rivas-Gutiérrez R, Díaz-Peña R (2013) Uso de microimplantesen el tratamiento de ortodoncia. Rev Tamé 2: 126-132. 